\title{
Análisis cualitativo de una propuesta pedagógica para la incorporación de entornos virtuales a la enseñanza universitaria en Argentina a partir de tres ejes: experiencia, rol docente y tiempo
}

\author{
A qualitative analysis of a pedagogical proposal for the incorporation of virtual \\ environments into higher education in argentina based on three axes: experience, teaching \\ role and time
}

\author{
Ana Borgobello ${ }^{1}$ \\ https://orcid.org/0000-0002-2340-8127 \\ Universidad Nacional de Rosario, Argentina \\ Sofía Majul ${ }^{2}$ \\ https://orcid.org/0000-0003-3006-530X \\ Damián De Seta ${ }^{3}$ \\ https://orcid.org/0000-0002-4020-2526
}

Instituto Rosario de Investigaciones en Ciencias de la Educación, Universidad Nacional de Rosario, Argentina

\section{Cita Recomendada}

Borgobello, A., Majul, S., \& De Seta, D. (2019). Análisis Cualitativo De Una Propuesta Pedagógica Para La Incorporación De Entornos Virtuales A La Enseñanza Universitaria En Argentina A Partir De Tres Ejes: Experiencia, Rol Docente y Tiempo. Hamut'ay, 6(3), 81-97.

http://dx.doi.org/10.21503/hamu.v6i3.1848

\section{RESUMEN}

Los estudios sobre formación docente en y con uso de entornos virtuales son numerosos en la literatura especializada, sin embargo, son escasos aquellos que abordan cómo narran los propios docentes su acercamiento a las TIC con miras a utilizarlas en la enseñanza. Por esta razón y a partir del dictado de un seminario de posgrado con forma taller de iniciación en el uso del entorno Moodle como complemento del aula regular de clases (bimodalidad, modalidad híbrida o blended learning), se analizaron cualitativamente las narraciones acerca de qué aprendizajes valoraron y qué dificultades tuvieron quienes aprobaron el mismo con el objetivo de problematizar tres ejes construidos dialécticamente desde la propuesta pedagógica y los trabajos finales: experiencia, rol docente y tiempo. Los resultados muestran ideas en torno a la experiencia del taller operando como agente de deconstrucción de ciertas tradiciones a partir del aprender haciendo; roles docentes que se relacionan con el uso mismo del entorno virtual y de la participación en el cursado como docentes de docentes y docentes con docentes; y el tiempo como favorecedor en procesos de aprendizaje y como apremiante

1 Investigadora Adjunta en CONICET, responsable del Proyecto "Los entornos virtuales como complemento del aula regular de clases (blended learning) en el nivel superior” y docente de "Metodologías de Investigación en Psicología” en la Universidad Nacional de Rosario, Argentina. borgobello@irice-conicet.gov.ar

2 Pasante de Iniciación Científica en el proyecto "Los entornos virtuales como complemento del aula regular de clases (blended learning) en el nivel superior", radicado en el Instituto Rosario de Investigaciones en Ciencias de la Educación (IRICE-CONICET/UNR)

3 Pasante de Iniciación Científica en el proyecto "Los entornos virtuales como complemento del aula regular de clases (blended learning) en el nivel superior", radicado en el Instituto Rosario de Investigaciones en Ciencias de la Educación (IRICE-CONICET/UNR) 
en términos de agenda y de dificultad del trabajo con colegas. Se concluye en la necesidad de investigar y de encontrar espacios de formación y encuentro en un contexto en el que, salvo experiencias aisladas con amplia trayectoria, el uso de aulas virtuales está basado en la voluntad individual y es aún incipiente y escasamente favorecedor.

Palabras Clave: Aulas virtuales; Nivel universitario; Formación docente; Aprender haciendo.

\begin{abstract}
Specialized literature on teacher training in and with the use of virtual environments is frequently found; however, there are just few papers that address how teachers state themselves their approach to ICT in order to use them in teaching. For this reason, and based on a postgraduate seminar called Introduction to the use of the Moodle environment as a complement of the regular classroom (bimodality, hybrid model or blended learning), we analyzed qualitatively the narratives about what teachers have learned and which difficulties they had when dealing with these technologies. The analysis aimed to problematize three axes constructed dialectically from the pedagogical proposal and the final projects: experience, teaching role and time. Results showed ideas related to the workshop experience as a deconstruction agent of certain traditions based on the premise learning by doing; teaching roles that relate to the use of the virtual environment itself and the participation in the course, as teachers of teachers and teachers with other teachers; and the time as a facilitator in learning processes and essential in terms of schedule and difficulty when working with colleagues. In conclusion, there is a need to research and find training and meeting spaces in a context in which, except for isolated experiences with extensive experience, the use of virtual classrooms is based on individual will and is still incipient and scarcely promising.
\end{abstract}

Keywords: Virtual classrooms; university level; teacher training; learning by doing.

\section{INTRODUCCIÓN}

La incorporación de tecnología no ha cambiado de manera sustantiva el esquema verticalista en el modo de transmisión de conocimientos ni ha constituido por sí misma un elemento transformador (Lorenzo, 2018). Las TIC (tecnologías de la información y la comunicación) en el escenario de la educación, considera Onrubia (2016), en ocasiones llegan incluso a reforzar modelos dominantes de enseñanza y aprendizaje, vale decir, el mero uso de TIC no implica innovación per se. En este sentido, según sostiene el autor, se debería dar relevancia a la investigación empírica que conlleve consideraciones específicamente educativas incluyendo el debate teórico de modelos que guían la incorporación de TIC. Desde esa perspectiva se sitúa el presente artículo, buscando poner en debate temas relativos al actual ingreso de TIC en un contexto de enseñanza universitaria tradicional que puede definirse como escasamente facilitador.

Una de las TIC formalmente en uso en el contexto local es Moodle. Ésta, al igual que otros entornos virtuales de enseñanza y aprendizaje (EVEA) o plataformas virtuales educativas, son, básicamente, aplicaciones informáticas diseñadas con fines pedagógicos (Szpiniak \& Sanz, 2009) y utilizadas como forma exclusiva de mediación con los estudiantes (e-learning) o en combinación con clases presenciales (diseños bimodales, mixtos, híbridos o blended learning). El propósito de estas radica en facilitar la comunicación entre los participantes de un proceso educativo y funcionan utilizando principalmente Internet. La 
plataforma Moodle, una de las más utilizadas en educación superior, tiene dos aspectos clave según Aragón (2013): usabilidad (es sencilla de usar), y contempla los contenidos didácticos en un encuadre pedagógico. Para Szpiniak \& Sanz (2009) el diseño tecnológico de los EVEA acompaña al modelo pedagógico favoreciendo los procesos educativos y poniendo énfasis en la interactividad como estrategia, aunque resulta necesario no perder de vista que la herramienta tecnológica por sí misma no garantiza el desarrollo de los procesos educativos. De hecho, Aragón (2013) plantea que la construcción de materiales para la enseñanza no es una cuestión técnica, sino que da cuenta de la relevancia de contenidos, lecturas, tareas, entre otros, que pueden determinarse sólo desde el conocimiento disciplinar y pedagógico y no desde los recursos tecnológicos únicamente. A pesar de ello, las posibilidades y limitaciones en la organización de la actividad conjunta relativa a los contenidos y tareas académicas, para Coll, Onrubia \& Mauri (2007), varían dependiendo de la naturaleza y características del equipamiento y recursos tecnológicos de los que se dispone. Además, el diseño tecnológico se encuentra acompañado de modo más o menos explícito (dependiendo de las instituciones) de sugerencias y orientaciones para utilizar esos recursos tecnológicos. En esta relación entre el diseño propiamente tecnológico y el marco institucional en el que se utiliza se encuentra el diseño tecnopedagógico. Si bien las normas y procedimientos de uso de las TIC son elementos esenciales del diseño tecnopedagógico, los autores recalcan que la organización de la actividad conjunta es consecuencia de la negociación y construcción basadas en expectativas, motivación, contexto, etc. de los participantes de un curso por lo que tampoco puede interpretarse como una mera traslación del diseño tecnopedagógico establecido institucionalmente.

Este artículo se centra en analizar las narraciones de docentes que tras finalizar un curso de formación inicial para el uso de Moodle realizaron escritos requeridos para aprobarlo. La línea de investigación en la que se inscribe este estudio tiene por objetivo general analizar las prácticas pedagógicas con incorporación de entornos virtuales en educación superior. Tiene un enfoque complejo dialéctico (Achilli, 2005) en el que se trabaja con datos tanto cualitativos como cuantitativos en el análisis de la interacción sociocognitiva del trabajo en el aula virtual como de sus condicionantes culturales. Si bien se ha trabajado con estudios cuasiexperimentales; basados en la etnografía virtual; de aplicación de modelos teóricos diversos al análisis de la interacción escrita; entre otros, el eje principal es el volver a pensar la clase (Sanjurjo \& Rodríguez, 2003, Sanjurjo, 2016) en sus condiciones materiales y discursivas.

A continuación, se describirán conceptos que las y los participantes han utilizado en sus escritos a partir de lecturas de literatura científica en una construcción espiralada. La misma comienza por el diálogo entre autores que dan cuenta de distintas maneras de los tres ejes de análisis propuestos -experiencias, rol docente y tiempo- para dar lugar a las palabras de quienes analizaron sus propios aprendizajes en el marco del mencionado seminario sobre Moodle. Por tanto, el objetivo del presente estudio fue analizar los escritos de los docentes en el marco del taller en relación con los tres ejes conceptuales expuestos.

\section{Sobre experiencias y roles docentes}

Los conceptos vinculados a experiencias docentes desde la incorporación de las TIC abrieron una diversidad de debates nuevos y renovaron discusiones sobre temas tradicionales en educación superior tales como dominio requerido de tecnologías en docencia; diversidad de fuentes de información; percepción de lo pedagógico en el uso específico de entornos virtuales; diferencias entre comunicación oral y escrita; formación docente; aprendizaje colaborativo; autonomía, guía, andamiaje y funciones tutoriales; entre otros.

Puede afirmarse categóricamente que quienes hoy son docentes fueron afiliados a las instituciones educativas (Pierella, 2017) en clases presenciales tradicionales y que, incluso, suelen enseñar de acuerdo a cómo aprendieron (Ventura, 2016). Sin embargo, en la actualidad, las experiencias en educación superior, al menos en modo declarativo, aparecen asociadas a un cambio profundo vinculado al uso de espacios virtuales y a la sociedad de la información que va desde el protagonismo del docente al del estudiante (Garrison \& Kanuka, 
Análisis cualitativo de una propuesta pedagógica para la incorporación de entornos virtuales a la enseńanza universitaria en Argentina a partir de tres ejes: experiencia, rol docente y tiempo

2004).

Un motivo de preocupación para algunos autores es que los estudiantes suelen vivir en la cultura de Internet y superar a sus docentes en el manejo de TIC y en la navegación que implica el acceso a información que circula por la red (Domingo \& Fuentes, 2010). García (2007) plantea que Internet como fuente de información es algo así como una vidriera en la que se pueden encontrar los más diversos materiales didácticos, experiencias y casos vinculados a las temáticas trabajadas en clases; en publicaciones con formatos diversos, entre otros aspectos. Sin embargo, sostiene, que se presentan dificultades en cuanto a las habilidades relativas a las estrategias y valoración en las búsquedas de información.

Otra de las preocupaciones percibidas por los docentes es la dificultad en el manejo técnico de la plataforma virtual en su implementación, mientras que lo específicamente pedagógico relacionado con el uso de entornos virtuales parece no ser una dificultad para los docentes (Borgobello, Sartori \& Sanjurjo, 2018). Adicionalmente, las diferencias entre la comunicación presencial y virtual son numerosas y suelen ser leídas por algunos docentes como conflictivas (Borgobello \& Sartori, 2016). Incluso las particularidades de interacción sociocognitiva de un mismo docente y grupo-clase en una clase dictada cara-a-cara y mediada muestran claras diferencias en los aspectos comunicativos en ambos espacios -tales como heterogeneidad discursiva del aula tradicional en comparación con la virtual (Borgobello, Sartori \& Roselli, 2016).

Estas ideas presentan una perspectiva distinta de la clásica separación basada en la experiencia entre novatos y expertos. Los conocimientos tácitos apropiados a partir de la experiencia, tales como los conocimientos pedagógicos, pueden resultar un obstáculo en la enseñanza. En este sentido, Pierella (2017) sugiere retornar a la inseguridad propia del novato (expresión válida para docentes y estudiantes) que ayudaría a orientar anticipando dificultades, destacando puntos conflictivos y subrayando cuestiones de relevancia -que no necesariamente se relacionarían con lo tecnológico. Estas formas de comprender la docencia como actividad reflexiva requieren formación y debates sostenidos en el tiempo. Acedo (2017) sostiene que en la enseńanza universitaria se ha dado escasa atención a la formación docente en comparación con otros niveles educativos y con otras funciones requeridas al profesorado.

El desafío, para Copertari, Sgreccia \& Fantasía (2011), sigue siendo que el docente en su calidad de experto facilite el desarrollo del estudiante para que logre conocimiento experto. En esta tarea se involucra tanto la tiza y el pizarrón como las TIC, aunque estas últimas cuentan con la ventaja diferencial de generar interactividad y flexibilidad entre estudiantes y profesores y una conectividad "cuasi-masiva".

Sotomayor (2016) recalca la importancia de distintas variables a ser tenidas en cuenta para llevar adelante experiencias colaborativas mediadas, a través del trabajo lógico del entorno Moodle. Una de ellas es de carácter pedagógico y está relacionada con el diseño de los ambientes colaborativos: se trata de establecer las condiciones que hacen posible la participación activa y productiva. Para ello se requiere contar con los recursos y andamiajes que fomenten los mecanismos de colaboración e indagación que generalmente no se dan de manera espontánea. Es decir, la autora considera que el aprendizaje colaborativo mediado (ACM) no se produce de forma automática, aplicándose por ello con poco éxito en los sistemas educativos formales.

Copertari et al. (2011) sostienen que la virtualización de la universidad es una necesidad basada en procesos técnicos, pero, esencialmente, son procesos políticos, sociales y culturales, lo que implica acciones estratégicas con énfasis en la formación docente dados los desafíos pedagógicos que implican. En este sentido, no debería basarse como ocurre a menudo en experiencias aisladas vinculadas al voluntariado de los docentes (Borgobello, 2018), sino en proyectos institucionales que faciliten transitar estos cambios.

Siguiendo a Silva (2012), quien menciona que hubo cambios en el rol docente a partir de la inserción de las TIC en la educación formal, lo que requiere un nuevo posicionamiento del profesor. La enseñanza pasaría a ser fundamentalmente 
guía y estar centrada en los estudiantes a partir de entornos que se propician como más interactivos y motivadores que las clases tradicionales, dejando de ser el docente fuente única de información.

Si bien la literatura sobre la idea de guía, andamiaje o funciones tutoriales es abundante, se advierte falta de consenso acerca de clasificaciones en torno a estos conceptos. A partir del análisis de distintas investigaciones, se propusieron categorías para pensar las mediaciones tutoriales en el nivel universitario que podrían aplicarse tanto a la interacción cara-a-cara como a la mediada digitalmente, tutores pares: con el mismo o con diferente grado de preparación; y expertos: orientadores o docentes (Borgobello \& Peralta, 2010).

Las funciones tutoriales entre pares suelen estar vinculadas a estudios sobre trabajo colaborativo grupal. Roselli (2016) alega a favor de la regulación externa del grupo en su gestión de la actividad académica. El docente, además de elaborar material didáctico y de organizar la composición de los grupos, debería regular la realización de la actividad y la interacción comunicativa entre estudiantes. Sostiene que se ha hecho un uso ingenuo del trabajo en equipo, malinterpretando conceptos provenientes del socioconstructivismo y del aprendizaje colaborativo. El aprendizaje de conocimientos epistémicos en situaciones formales necesitaría pre-requisitos o condiciones que hacen posible un real y eficaz trabajo colectivo.

\section{Sobre el tiempo y los recursos mediadores}

De acuerdo con la experiencia, a estudios previos y a la literatura específica, encontramos que el tiempo, tanto como categoría objetiva como en relación a la experiencia subjetiva, es para los docentes un fuerte determinante de las experiencias laborales.

García (2007) sugiere que las novedades que introdujeron las TIC en el campo laboral de la docencia requieren mayor dedicación en tiempo y esfuerzo como así también capacidades y destrezas diferentes a las tradicionales. Montero \& Gewerc (2010) plantean que las instituciones educativas experimentan una situación dilemática entre la conservación de aspectos culturales reconocidos y las demandas hacia la innovación dando lugar a un clima de agobio asociado a la falta de tiempo del cuerpo docente para pensar, reflexionar, practicar y evaluar eventuales cambios utilizando TIC. Asociado a esta falta de tiempo reflexivo se encuentra lo que García (2007) menciona: al docente se le presenta trabajo añadido en planificación, seguimiento y evaluación al incorporar entornos digitales; y el estudiante, a su vez, requiere más tiempo para el seguimiento regular de la asignatura con estos cambios. Por otro lado, señala, las plataformas digitales aportan a la organización de actividades realizadas online relativas al desarrollo de una asignatura, estructurando no solo tiempos sino también temas y recursos. Los recursos que ofrecen son diversos y facilitan la evaluación del trabajo de los estudiantes, permitiendo hacer un seguimiento individualizado o de grupos pequeños. Asimismo, posibilitan potenciar el trabajo colaborativo, el aprendizaje autónomo y la responsabilidad del estudiante en su implicación en el estudio.

Una de las razones por las que los participantes en entornos virtuales presentan resistencia al cambio es porque, según Aragón (2013), suelen estar acostumbrados a docentes que otorgan conocimiento y la modalidad híbrida requiere una actitud participativa y crítica de los y las estudiantes. Cabe señalar que suele haber diversos perfiles en cuanto al uso de los entornos digitales, aunque como se dijo, se encuentran limitados de acuerdo a la disponibilidad tecnológica (Coll et al, 2007). Se distinguieron tres perfiles de estudiantes relacionados con el uso de la plataforma (Borgobello \& Roselli, 2016). El primero de ellos, se destacó por el escaso uso del entorno virtual y por la preferencia en el uso de foros, por lo que podría entenderse como el recurso más intuitivo. El segundo grupo, el mayoritario, mostró alta aceptación al uso de Moodle, usó la plataforma fundamentalmente en cercanía al examen parcial, se caracterizó por preferir cuestionarios, siendo este el recurso menos interactivo propuesto. Quienes más utilizaron el entorno integraron el tercer grupo que no se caracterizó por preferir algún recurso en particular. Este último grupo se destacó por considerar a las explicaciones docentes presenciales como claras, lo que indicaría que quienes más utilizan el espacio virtual, comprenderían mejor 
Análisis cualitativo de una propuesta pedagógica para la incorporación de entornos virtuales a la enseñanza universitaria en Argentina a partir de tres ejes: experiencia, rol docente y tiempo

el contenido académico. Además, quienes tuvieron más participación, obtuvieron mejores calificaciones, les agradó más utilizar el espacio virtual y escribieron más cantidad de mensajes en foros.

Uno de los recursos de Moodle más versátiles y utilizados por los docentes universitarios son los foros que constituyen el principal canal de comunicación en este nivel (Lorenzo, 2018). Ezeiza \& Palacios (2009) destacan que la competencia comunicativa y social de los estudiantes evoluciona en los foros virtuales al ser utilizados como complemento de las clases presenciales.

Los estudiantes con mayor desempeño académico, además de dedicar más tiempo al uso de la plataforma, tendrían mayor compromiso con las y los demás y más apropiación del espacio virtual de interacción. Esto puede afirmarse dado que los estudiantes con más alto rendimiento académico integral suelen escribir con más contenido socioafectivo y anidan sus mensajes en los foros (dan respuestas y reciben posteos de otros estudiantes), además de escribir en foros optativos y usar primera persona en la redacción de sus respuestas (Borgobello \& Roselli, 2016). Asimismo, no solo el recurso sino también el tipo de tarea (como por ejemplo lógico-cerradas o libres y hermenéuticas) determinaría las características de interacción colaborativa en espacios digitales (Roselli, Dominino \& Peralta, 2010).

Más allá de los recursos y tareas específicas, la experiencia discursiva en las comunidades virtuales presenta distintas dimensiones que requerirían cierto balance. Garrison (2007) sostiene que esta se encuentra conformada por la presencia cognitiva, social y docente. Lo cognitivo refiere al desarrollo progresivo desde la exploración hasta la resolución en cuanto al contenido; la presencia social está constituida por expresiones de tipo motivacionales que propician el apoyo socioemocional que cohesiona al grupo; $y$ la presencia docente es considerada como el diseño, facilitación e instrucción directa. Cuando el diseño pedagógico es bimodal, la presencia social puede ser más escasa que cuando se produce exclusivamente en línea. Esto podría deberse a que la interacción cara a cara daría acuerdos previos dejando la interacción virtual basada exclusiva o predominantemente en la tarea académica (Borgobello et al, 2016).

En el ámbito local, la tendencia en las ofertas educativas responde a la bimodalidad basada principalmente en el Campus Virtual (Moodle) más que al desarrollo del cursado exclusivamente en línea (Copertari, Sgreccia \& Fantasía, 2010).

Alucin (2018) remarca que la incorporación de las TIC propicia cambios pedagógicos que redefinen al aula tradicional, dando lugar a su potencialidad democratizadora, ya que permiten la consolidación de espacios públicos virtuales, accesibilidad al conocimiento, posibilidad de dinámicas colaborativas para educar e investigar, llegando, a su vez, a públicos diversos más allá de la disponibilidad espacial y temporal. La autora señala que, si bien las políticas de virtualización en el contexto local han avanzado de forma considerable, aún puede considerarse escasa la implementación de espacios virtuales. Por otra parte, y posiblemente relacionado con esto último, Lorenzo (2018) señala que cuando el soporte técnico es malo y no funciona bien, los docentes tienden a desestimar el uso de las TIC. Alucin (2018) enfatiza que la continuidad en el avance en la incorporación de TIC en el ámbito educativo local requiere investigación orientada a la acción que pueda realizar aportes reconociendo experiencias, fortalezas y dificultades.

\section{Materiales y Métodos}

\section{Participantes}

La población en estudio lo conformaron inicialmente 14 estudiantes del taller dictado en el 2018 sobre Blended learning en una universidad pública argentina, con predominancia de interacción en el entorno virtual Moodle con una única clase presencial de cuatro horas realizada promediando el cursado. Todos los participantes eran docentes de grado con edades de entre 27 y 62 ańos, con formación en pregrado y posgrado heterogéneas (Psicología, Ingeniería, Ciencias de la Educación y Filosofía). Se consideró para esta investigación el trabajo conjunto en el taller y como muestra intencional (Echevarría, 2016) los ocho trabajos finales presentados por los y las estudiantes que aprobaron el taller (identificados como D1 a D8). 
La selección del material y la muestra buscó evitar sesgos vinculados, por ejemplo, a la escasa motivación o dedicación relativas al curso.

\section{Tipo y diseño de estudio}

El tipo de estudio es cualitativo y el diseño descriptivo (Echevarría, 2016), ya que se realizó una descripción a través de las narraciones de los docentes que formaban parte de sus trabajos escritos aprobados en el marco del taller desde tres grandes ejes construidos desde la propuesta pedagógica y desde las narraciones: experiencia, rol docente y tiempo. Se enfatizó tanto en las repeticiones terminológicas o conceptuales como en decires excepcionales que generaran atención a partir de las particularidades del caso.

\section{Instrumento}

Para analizar las narraciones de los estudiantes se aplicó análisis de contenido cualitativo (Piovani, 2007) a las respuestas dadas a dos consignas del trabajo final: (a)- Aprendizajes más relevantes logrados en relación con la propuesta pedagógica general y (b)- Dificultades encontradas en el proceso general de cursado relacionadas con las exigencias del taller. El trabajo final a presentar incluía otra consigna (que no fue analizada en este estudio por razones de espacio) que requería el diseño pedagógico de una actividad que incluyera virtualidad y presencialidad factible de ser aplicada a los estudiantes. Las narraciones analizadas fueron extraídas de los trabajos finales solicitados para la aprobación del Seminario de Posgrado con formato de taller

\section{Procedimiento}

El procedimiento de construcción de los datos (Figura 1) implicó el análisis cualitativo de las narraciones escritas en los trabajos finales acerca de aprendizajes valorados y dificultades encontradas por los participantes del taller. Tres investigadores leyeron los textos en forma independiente. A partir de esa primera lectura se constituyeron los tres ejes de análisis desde la relevancia otorgada por los participantes a esas ideas: experiencia, rol docente y tiempo.
La construcción de los ejes implicó sucesivas lecturas de los trabajos. Mientras que "rol docente" y "experiencias" formaron parte de los conceptos trabajados en las clases, el eje "tiempo" fue surgiendo como problemática emergente. Cabe señalar que, como es de esperar, hubo otros numerosos conceptos trabajados en el taller que en los escritos no fueron destacados, por ello, solo se tuvo en cuenta estos dos conceptos y que se constituyeron en ejes y material de análisis.

Siguiendo el enfoque cualitativo, se respetó la singularidad por lo que se destacó en los resultados la letra propia, la narrativa, la forma en que quienes participaron de la investigación escribieron.

Una vez explicitados los ejes, se procedió a extraer del material cada párrafo en el que se mencionaran los mismos construyéndose una matriz cualitativa. A partir de los tres ejes se procedió a nuevas lecturas conceptuales y a la síntesis de los datos en base a criterios interpretativos y contextualizados tanto en el marco del propio seminario como de la universidad en la que se dictó el mismo. Los resultados presentan una síntesis de esas interpretaciones basadas en las sucesivas lecturas realizadas por el equipo de trabajo.

El objetivo, como se ha mencionado, se centró en problematizar los tres ejes construidos en forma dialéctica desde la propuesta pedagógica del taller y los trabajos finales entregados al finalizar el mismo. Por ello, la organización del taller y el dictado de este fueron progresivamente resignificados a partir de la presentación de trabajos finales constituyéndose en objetos de análisis.

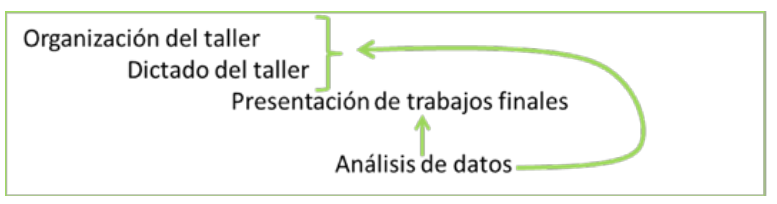

Figura 1. Fases progresivas de trabajo didáctico y análisis de datos.

Fuente: Elaboración propia (2019)

\section{Consentimiento informado}

Se resguarda el anonimato de las y los participantes identificándose a cada uno con la letra D y 
un número para facilitar la interpretación de los datos. Cada participante firmó un consentimiento informado siguiendo los protocolos de ética vigentes en la universidad en la que fue radicado el proyecto.

\section{Resultados}

La organización de los resultados obtenidos responde al objetivo del presente estudio que radicó en analizar los escritos de los y las docentes en el marco del taller mencionado en relación con tres ejes conceptuales propuestos: experiencias, rol docente y tiempo.

Experiencias: inicios, continuidades y discontinuidades

En este apartado se destacan desde las narraciones la experiencia del taller; el conjunto de habilidades y competencias previas y adquiridas durante el cursado; como así también hitos de las biografías y trayectorias singulares señaladas por las y los participantes. A partir del análisis de los trabajos escritos en relación con los aprendizajes logrados y las dificultades encontradas durante la realización del taller, extrajimos los decires relacionados con la idea de experiencia/s que emergían de los datos.

Algunos docentes destacaron la apropiación de procesos instrumentales vinculados a las herramientas y recursos disponibles en la plataforma: "El curso me ha permitido iniciarme en un campo para mi absolutamente nuevo, explorar sus recursos $y$ modalidad de uso, generando en mí mucho interés durante su realización" (SIC, D2). Generalmente esta forma de conceptualizar la experiencia respondió a docentes que se iniciaban en el uso de Moodle con este taller tal como puede percibirse en el ejemplo extraído de uno de los trabajos.

En relación con la experiencia particular de cursado, fue recurrente encontrar descritos como aprendizajes relativos al taller una lista de competencias o habilidades. En algunos casos, se describían recursos puntuales relacionados con habilidades de la informática en general además de Moodle, por ejemplo: realizar capturas de pantallas, cortar y pegar utilizando el teclado, buscar imágenes en internet, copiar hipervínculos, reducir el peso de archivos para adjuntar, entre otros. Varias personas enumeraron en estas listas recursos particulares del aula virtual que podrían trabajar luego en sus propios diseños pedagógicos: "Aprendi acerca de nuevos recursos como la construcción del glosario, las modalidades de los procesos evaluativos, el manejo de las imágenes y/o videos, entre otros"(SIC, D8).

En los escritos presentados se destacó que la experiencia de trabajo con los recursos en un entorno como Moodle responde a una lógica, específicamente la del aprendizaje colaborativo: "En las primeras actividades me llevó más tiempo entender la lógica de la plataforma Moodle, pero a medida que fuimos avanzando (...) fui logrando situarme y aprovechar los recursos disponibles para cada actividad" (SIC, D2). Al revelar la existencia de una lógica subyacente en la plataforma virtual que requiere familiarizarse con la misma, una docente reconoció la imposibilidad de abarcar la totalidad de las herramientas y recursos: "Si bien no se abarcó la totalidad de las posibilidades (son demasiado amplias para ser abarcadas en una cursada), logré una familiaridad con la herramienta que me permite comprender la lógica con la que se trabaja y me da la posibilidad de experimentar teniendo noción de los alcances y las posibilidades que se ofrecen" (SIC, D7). Si bien retomaremos esto luego, cabe adelantar que la familiaridad con los entornos virtuales y su lógica desde el rol docente, tal como lo expresaron en los escritos, requiere tiempo de trabajo en esos espacios.

En cuanto al formato de los recursos que tiene Moodle cabe destacar que los foros pueden diseñarse de diversas formas, siendo, probablemente, la más común la que genera anidaciones diversas a partir de que un o una participante ańade un nuevo tema de discusión (presionando en "Añadir nuevo tema de discusión"). Para poder leer las réplicas o respuestas a ese tema se requiere entrar en el vínculo al que el participante puso un título determinado: "En referencia a otras prácticas de formación a través de plataformas que he realizado, la existencia de foros con varias aperturas me generó al inicio cierta confusión, pero luego fui encontrando 
la lógica propia del modo que se nos habia propuesto para el intercambio"(SIC, D1). Aquí nuevamente, se manifestó la idea de lógica del taller que se vincula estrechamente con la lógica de la plataforma, pero esta vez en términos de una experiencia cargada de dificultad o confusión a partir de conocer otras. Cabe señalar que, cuando los diálogos fueron fluidos, las conversaciones derivaban hacia temas diversos a la inicial, quedando vinculados a un tópico que no necesariamente era claro. $\mathrm{Si}$ bien es posible buscar a partir de una palabra en todos los foros, esto, en la experiencia compartida, no pareció utilizarse.

En relación a lo dicho, una de las críticas que se manifestaron entre los y las docentes fue que Moodle, como entorno virtual, era poco intuitivo: "La plataforma Moodle, a pesar de que vengo trabajando hace un par de años con ella desde mi práctica docente en la facultad, tiene la característica de amplitud de herramientas, lo cual presenta, a mi criterio, la dificultad de alejarse del uso intuitivo con que cuentan otros formatos virtuales. Se hace imprescindible su uso constante, de manera de familiarizarse en el tiempo con la misma" (SIC, D5). Esto que para algunas personas resultó una ventaja de esta TIC, para otros, como puede advertirse en este caso, representó un obstáculo.

$\mathrm{Al}$ igual que la docente citada anteriormente, otro participante del taller con experiencia previa en el uso de aulas virtuales como complemento del aula tradicional, destacó la importancia de basar las decisiones fundamentadas en criterios pedagógicos como parte del aprendizaje del taller: "Durante el cursado del Seminario conocí algunas herramientas que la plataforma Moodle proporciona para llevar adelante experiencias de enseñanza y aprendizaje. (...) Si bien estaba familiarizado con la plataforma Moodle, el seminario funcionó como estimulante para abordar las herramientas desde otras perspectivas, dando sustento a las decisiones al momento de elegir las más adecuadas según las necesidades"(SIC, D7).

Consideramos que, a partir de lo dicho por los participantes, la experiencia de encontrarse con otros docentes para discutir y reflexionar sobre prácticas pedagógicas implicó un estímulo para pensar y repensar la docencia. Una de las parti- cipantes resaltó como innovación compartir un espacio sobre docencia con colegas de otras asignaturas o disciplinas, algo visto aparentemente como poco frecuente en la educación superior: "...una novedad empezar a discutir en foros y chats permanentes opiniones, experiencias de distintos ámbitos"(SIC, D4).

Cada participante manifiestamente inscribió el taller como hito en su propia trayectoria experiencial: "los cursantes hacemos un recorrido singular, en el que juegan las propias biografias académicas y prácticas" (SIC, D5). Estas formas de entrar al uso de entornos virtuales respondieron no sólo a expectativas sino también a prejuicios, tal como se expresa en la siguiente afirmación: "Una de las dificultades fue confrontarme con mi escasa experiencia previa en el uso de este tipo de plataformas de aprendizajes $y$, quizás, cierto prejuicio personal acerca del uso de las mismas, propiciado en parte por algunos criterios que presentan el uso de las TIC (...) como una garantía de cambio en las relaciones con la construcción del conocimiento" (SIC, D2). Así, podemos destacar que como parte del cursado se acentuó el reconocimiento de contextos y diseños pedagógicos, descartando la idea de que las TIC son, por ejemplo, motivadoras por sí mismas.

En el mismo sentido, durante el cursado se propiciaron discusiones entre docentes en las que las experiencias de evaluación generaron amplios debates, poniendo en tensión los criterios pedagógicos predominantes en las universidades en estos temas. Se marcó a la evaluación como inserta en el espacio de la plataforma en diseños tecnopedagógicos dados en contextos educativos siempre particulares: "las cuestiones acerca de la evaluación fueron muy espinosas. Estas últimas cuestiones acerca de la evaluación me resultaron muy interesantes porque aprendi que en este entornolámbito evaluar significa poner en juego los aspectos más jugosos o novedosos de la inclusión de una plataforma en la enseñanza universitaria: ¿Qué teoría de la enseñanza y el aprendizaje sostenemos cuando estamos en la Universidad?, ¿Cómo ésta condiciona mi relación al uso o no que hago de la plataforma?"(SIC, D3).

A modo de cierre de este apartado, se propone destacar desde las narraciones el concepto de aprendizaje en un aprender haciendo, la experiencia 
Análisis cualitativo de una propuesta pedagógica para la incorporación de entornos virtuales a la enseñanza universitaria en Argentina a partir de tres ejes: experiencia, rol docente y tiempo

como actitud de exploración reflexiva, así como lo expresó una participante: "Uno de los aprendizajes más relevantes que puedo mencionar es el registro del impacto que puede tener la lógica de "aprender haciendo". (...) lo más significativo como experiencia de aprendizaje fue el poder experimentar, ensayar o poner a prueb a esos conocimientos"(SIC, D1). Otra participante planteó: "Este aprender haciendo me recuerda el concepto de conocimiento inactivo propuesto, entre otros, por Bruner, para reconocer un modo posible de conocimiento, "un camino de organización de conocimiento", un aprender haciendo cosas (Guillar 2009)" (SIC, D8). Sintéticamente, el concepto de aprendizaje propuesto en esta última afirmación se diferencia de la idea de ensayo y error en el sentido de proceso, de continuidad, en un aprender haciendo, experimentando, ensayando y poniendo a prueba.

Roles docentes: docentes de docentes y docentes con docentes

En este apartado el eje de análisis rol docente se relacionó con distintas ideas, algunas vinculadas a roles reconocidos tradicionalmente en la universidad y otras asociadas a los sistemas digitales, como usuarios diferenciados a partir de distintas asignaciones de edición.

En cuanto a la docencia universitaria, la idea de rol docente quedó vinculada en numerosas oportunidades a complementar la cursada tradicional con la incorporación de entornos virtuales, denominada también, como dijimos, blended learning. Así, una de las participantes expresó estos conceptos del siguiente modo: "Este reconocimiento [del espacio y sus posibilidades pedagógicas] para mi es fundamental a la hora de la construcción de un espacio propio como docentes. Es el comienzo del recorrido que nos permitirá incorporar esta herramienta como colaboración a las clases presenciales" (SIC, D6). En el marco de este escrito, es interesante destacar la idea de colaboración como aporte del espacio virtual al presencial cuando se encuentra a cargo de un mismo docente. Otra participante también destacó la idea de hibridación entre escenarios como una de las riquezas del taller: " $E l$ curso me posibilitó reflexionar y pensar las actividades docentes combinando espacios virtuales junto con instancias de enseñanza presenciales, encuentro en esta articulación una de las mayores riquezas de la propuesta, entre la distancia y la presencia (...) Blended learning" (SIC, D8).

En numerosos escritos el rol docente universitario quedó asociado al aula tradicional, constituyéndose, incluso, como reclamo. La presencialidad o necesidad de encuentros pedagógicos cara-a-cara estuvo en casi todos los escritos de diferentes maneras. Cabe señalar, como dijimos en apartados anteriores, que la universidad en la que se dictó este taller, aunque con excepciones, tiene una fuerte tradición presencial. Por ejemplo, uno de los participantes reclamó de algún modo mayor tiempo de encuentros presenciales en la estructura del taller: "Una sola clase presencial me pareció insuficiente" (SIC, D4). Si bien hubo demanda para aumentar la cantidad de encuentros presenciales, también nos encontramos con una clara dificultad organizativa relacionada con la diversidad de agenda de los participantes. Finalmente, y a modo de solución de compromiso, se ofreció la posibilidad de encuentros presenciales opcionales que tuvieron, por lo dicho, escasa participación.

Las diferencias entre las formas de enseñanza presencial y virtual, en términos de comunicación, fueron leídas por algunos participantes como conflictivos: "La diferencia de comunicación entre el espacio virtual y el presencial, aunque he realizado formaciones online me sigue generando dificultades. El contexto de sentido y la consensuación que da el entorno presencial no lo tiene o es diferente en el entorno virtual. Esta dificultad, a mi entender, se vio agravada por mi imposibilidad de asistir a la clase presencial" (SIC, D6). Aquí la presencia fue leída como encuentro cara-a-cara, dándose sentido y consenso a partir del aquí y ahora.

Adicionalmente, el rol docente fue expresado como conjunto de permisos de edición, tal como se define el profesor dentro de un sistema como Moodle. Para comprender las narraciones de quienes participaron en el cursado, se hace necesario realizar algunas aclaraciones respecto a la propuesta pedagógica que tuvo lugar. Se implementaron dos espacios o cursos diferenciados en el mismo campus: en uno de ellos, las docentes a cargo del taller tenían rol profesor y los participantes tenían 
rol estudiante, mientras que en otro curso los participantes podían editar teniendo asignado el rol profesor en ese curso. A este último lo denominamos Zona de Prácticas y al primero, simplemente Taller de Moodle. A modo de familiarización con la plataforma, durante las primeras semanas solo se trabajaba en el espacio Taller implementándose el de las prácticas una vez avanzado el cursado.

La labor docente en el diseño y ejecución del curso puede leerse en la siguiente narración: "De manera particular me interesó que, comprendi y hallé muy novedoso el lenguaje, la disposición personal, el trato colaborativo y alentador-necesario y requisito imprescindible-para establecer un vinculo interactivo y constructivo en el proceso de aprendizaje virtual. Esto lo experimenté en la ejercitación y en la práctica misma con la plataforma" (SIC, el destacado es de la participante D8).

La práctica en el ejercicio del rol docente en términos de la especificidad de lo digital, uno de los ejes de trabajo del taller, fue señalada por los participantes: "...la zona de prácticas nos permitía ubicarnos desde nuestro lugar de profesores" (SIC, D1); o, como lo expresó otra participante: "empezar a operar en la plataforma como docentes" (SIC, D3). En este sentido, el rol docente aparecía como una cierta aplicación posible, desde la "exigencia de pensarlo en una situación real de enseñanza, lo cual nos puso en un contexto concreto para implementar el entorno virtual como espacio de enseñanza" (SIC, D1).

Los entornos virtuales basados en el concepto de aprendizaje colaborativo generan oportunidades de diálogo que ponen en juego el conocimiento como una construcción colectiva. Este concepto de aprendizaje colaborativo, dada la importancia que tiene específicamente en relación a Moodle, fue uno de los ejes que se trabajaron en el taller, destacándose por parte de los participantes la importancia del intercambio de ideas, conceptos y experiencias. Así apareció la imagen de docentes con otros docentes en términos de colegas que fue desarrollándose e incrementándose durante la cursada: "Fue muy provechoso el intercambio con los demás docentes, conocer sus experiencias e inquietudes. La variedad de situaciones y casos expuestos y las resoluciones particulares compartidas en los foros revelan distintas visiones que enriquecen la labor docente" (SIC, D7). Conocer otras experiencias, desde este decir, enriquecería a partir de socializar perspectivas diversas.

En relación a lo expuesto, una docente destacó la función tutorial como docente de docentes y el aprendizaje colaborativo como función tutorial par, pudiéndose dar ambas al mismo tiempo: "Las dificultades que encontré fueron subsanadas satisfactoriamente en el propio contexto del curso y con la colaboración permanente de las docentes y los aportes de las y los compañeros/as" (SIC, D8).

Sintéticamente, las docentes que coordinaron el curso actuaron como docentes de docentes, en el sentido de organizar los contenidos, elaborar un programa de un curso de posgrado y reconocerse en una propuesta pedagógica en la que otros docentes jugaban el rol de estudiantes. Sin embargo, y fundamentalmente a medida que avanzaba la cursada, los y las colegas generaron la paulatina distribución del rol docente entre participantes, según las expectativas de la propuesta pedagógica.

Finalmente, el curso se propuso como destinado a docentes en ejercicio, por lo que las propias prácticas pedagógicas de los cursantes estuvieron presentes como experiencias y como diseńos tecnopedagógicos a futuro a partir del inicio del uso de Moodle como entorno virtual acompañando las clases presenciales. Por esta razón, la cursada dio lugar a particularidades que destacaron la necesidad de adaptaciones posibles a las estructuras de cursado, mostrando diversidad de roles docentes: "Las dificultades que se me presentaron no tuvieron que ver tanto con el desarrollo del seminario y los contenidos abordados (a mi entender fueron claras las consignas y excelente el apoyo para cumplirlas) sino con limitaciones personales en cuanto a mi formación y la práctica docente que desempeño en mi puesto de trabajo. El seminario está orientado (aunque no fue excluyente) a docentes que se desempeñan en cátedras en carreras de grado y mi labor se desarrolla (...), apoyando a los docentes. De todas maneras, recibi buenas sugerencias para adaptar las actividades propuestas a mi situación particular" (SIC, D7). Esto que la docente lee como limitaciones personales puede ser entendido también como parte de la diversidad de roles docentes que 
Análisis cualitativo de una propuesta pedagógica para la incorporación de entornos virtuales a la enseńanza universitaria en Argentina a partir de tres ejes: experiencia, rol docente y tiempo

encontramos en las instituciones educativas.

\section{El tiempo como obstáculo y como posibilitador}

Este último eje de análisis, el tiempo como obstáculo y como posibilitador, tiene la particularidad de haber sido elaborado a partir de las mismas narraciones expuestas más arriba como así también de la experiencia general del cursado del Taller. Se encuentra vinculado, tal como se expone a continuación, con los dos ejes anteriores: experiencia y rol docente. La idea de tiempo y docencia atravesó las narraciones de manera sistemática justificando así un apartado dada su predominancia en los resultados.

El tiempo en cuanto a la idea de experiencia en el marco del taller, apareció en los escritos en la enumeración de actividades y en la comprensión en etapas de, por ejemplo, la comprensión de la lógica de la plataforma. Estas etapas estuvieron signadas por el diseño tecnopedagógico del curso que progresivamente abría posibilidades de edición al incorporar la Zona de Prácticas (en la que los participantes podían editar como profesores) luego de haber participado únicamente como estudiantes (rol que permite escasa edición) al iniciarse este.

Los y las cursantes destacaron el acompañamiento de las docentes responsables del taller como así también de los compañeros y las compañeras de cursado en el aprendizaje colaborativo y la socialización de experiencias. En las narraciones puede leerse cómo la presencia docente fue distribuyéndose paulatinamente desde la demanda casi exclusiva a las coordinadoras del espacio hacia sus colegas. Esa distribución respondió a consignas que vinculaban a los participantes entre sí cada vez con más frecuencia a medida que avanzaba el cursado, propiciando de ese modo un clima áulico generado a partir de la fluidez respetuosa de intercambio de experiencias y saberes.

Desde la idea de familiarizarse con los recursos y con la lógica de Moodle, el tiempo fue leído como un transcurrir, por un lado, y como una suma de experiencias parciales, por el otro. En este sentido, las y los docentes mencionaron la posibilidad de exposición e intervención en los espacios virtua- les, la ejercitación con y en el espacio virtual, en un aprender haciendo. La familiarización con las herramientas y recursos se asoció en los escritos al aprendizaje como conjunto de procesos resignificados y como práctica reflexiva. Se podría decir que la iniciación en el uso de espacios digitales en forma complementaria al aula tradicional de clases llevó a repensar aspectos cruciales del aula tradicional, de los encuentros cara-a-cara que venían sosteniendo desde sus inicios en la docencia (y quizá antes, ya que en gran medida enseńamos como aprendemos). Al trabajar reflexivamente en cómo incorporar herramientas distintas a las que venían utilizando, la práctica cotidiana de enseñanza, según lo expresaron, se volvió un foco más claro.

Se mencionó que resulta más simple y rápido aprender en términos discursivos desde perspectivas en las que predomina lo expositivo. Sin embargo, se subrayó el aprender haciendo como modalidad de trabajo y de aprendizaje, aunque demanda más recursos y requiere, por tanto, más tiempo. Si bien fue algo destacado como positivo en los escritos finales, también se manifestó que aprender desde un rol activo resulta algo agobiante por momentos.

La novedad como disrupción en el tiempo pudo asociarse a la incorporación (o resignificación) del léxico técnico propio de estos espacios que implicó leer y releer algunos vocablos en numerosas oportunidades a modo de apropiación en la experiencia de encuentros significativos con esas palabras. La apropiación de vocabulario específico para sostener diálogos sobre lo pedagógico con uso de TIC formó parte, probablemente, de la familiarización con los recursos y la lógica de la plataforma de la que hablamos más arriba.

En el sentido de continuidad, el tiempo fue pensado desde la necesidad de regularidad en las entradas al espacio virtual como posibilidad de seguimiento de los diálogos. En los escritos se mencionó que la fluidez en los intercambios de ideas se veía condicionada por el hecho de entrar al espacio digital al menos dos veces cada semana ya que, cuando no lo hacían, resultaba algo agotadora la lectura de la cantidad de posteos que se producían o perdían alguna información o tema 
de interés hasta que alguien más lo retomaba. Aquí se esboza el formato de diálogo propio de lo asincrónico, categoría vinculada al intercambio en el cursado como tiempos discontinuos.

En numerosos escritos el tiempo se vinculó a la idea de agenda, de diagramación del tiempo, a veces, como se dijo, generando algo de agobio. Por un lado, la agenda respondía a entregas parciales a ser evaluadas, unidades de trabajo del programa del taller que se iniciaban y terminaban, es decir, trabajar según el tiempo de otras personas (en este caso, las coordinadoras del curso). Por otro lado, la agenda del curso se insertó en agendas particulares de cada participante, por lo que se volvió disponibilidad de tiempo para seguir una agenda compartida con un grupo.

Cabe señalar adicionalmente que el requerimiento formal de actividades propias de la agenda del curso implicó, en numerosas ocasiones, una mayor dedicación de la estrictamente necesaria dado que se volvió algo lúdico. En la construcción de actividades hipotéticamente pensadas para sus estudiantes, la motivación llevó al disfrute en construcciones creativas, cargadas de imágenes, videos, colores que implicaron navegación por diversos espacios, producciones $y$, por consiguiente, tiempo. Cabe señalar que el taller era optativo, por lo que el solo hecho de hacerlo se asoció a pensar formas de mejorar la propia docencia desde la dedicación voluntaria de tiempo.

\section{Discusión y Conclusiones}

En un contexto escasamente favorecedor de la incorporación de TIC en la enseñanza superior, se analizaron los escritos de docentes que aprobaron un curso para iniciarse en el uso de Moodle como complemento del aula tradicional de clases. Es en ese entramado de estrategias pedagógicas en las que pueden comprenderse los análisis realizados desde los tres ejes propuestos: experiencia, rol docente y tiempo.

Se buscó que la experiencia del taller, en el primer eje de análisis del presente escrito, pudiera hacer huella en la experiencia docente de quienes realizaron el curso. Este énfasis pudo observarse en las narraciones dadas en los trabajos finales a partir de la invitación a escribir acerca de los aprendizajes logrados y las dificultades encontradas desde la propuesta pedagógica. En este sentido, se logró desdibujar las ideas de novatos y expertos, horizontalizando la enseñanza y dando lugar a un progresivo trabajo colaborativo y socialización en el grupo que iba reconociendo diversas novateces y experticias dependiendo de los diálogos que tuvieran lugar (Copertari et al, 2011; Domingo \& Fuentes, 2010, Sotomayor, 2016). Así, tal como reconocieron en los escritos, el protagonismo pasó desde estar centrado en las coordinadoras a estarlo en las y los participantes, algo propiciado por los entornos virtuales diseñados para el trabajo en educación (Garrison \& Kanuka, 2004; Sotomayor, 2016; Roselli, 2016).

El taller funcionó como caja de resonancia de la propia trayectoria docente, generando expectativas al iniciar el uso de entornos virtuales en docencia, como también operando en la desnaturalización de prejuicios como pensar que el uso de TIC puede funcionar como garantía de cambio o de motivación por sí misma (Aragón, 2013; Borgobello et al, 2018; Onrubia, 2016).

En este mismo eje de análisis, además, se destacaron en las narraciones la idea de experiencia y de experiencias, es decir, por un lado, en singular y refiriendo a biografías y trayectorias como estudiantes y docentes singulares; y, por otro lado, en plural dando idea de un conjunto de habilidades y competencias como posibilidad de uso de herramientas y recursos disponibles en la plataforma. Cabe destacar que la habilidad en el manejo técnico de la plataforma virtual es una preocupación docente recurrente (Borgobello et al, 2018). Esta última idea quedó asociada a la exploración de posibilidades como novedad y estuvo presente fundamentalmente en quienes con este taller se iniciaban en el uso de aulas virtuales.

Los trabajos presentaron en forma recurrente la idea de lógica de la plataforma basada en el trabajo colaborativo (Sotomayor, 2016) que requiere familiarizarse con la misma para comprender cómo utilizarla. Aquí cabe preguntarse en qué modo afecta la idea de tecnología como aplicación de lo que otros diseñan para que los docen- 
Análisis cualitativo de una propuesta pedagógica para la incorporación de entornos virtuales a la enseñanza universitaria en Argentina a partir de tres ejes: experiencia, rol docente y tiempo

tes usen. Es pertinente señalar que esta familiarización fue entendida tanto como la habilidad de uso (García, 2007) que facilita su aplicación y como dificultad.

En relación a las experiencias con los recursos en su lógica, el foro se constituyó como tema recurrente en los escritos. Fue uno de los recursos más utilizados en el taller dada su versatilidad (Lorenzo, 2018) y por ser de preferencia de quienes utilizan escasamente los espacios virtuales ya que podría entenderse como uno de los recursos más intuitivos (Borgobello \& Roselli, 2016). Al utilizarse de modo frecuente multiplicando los diálogos, como recurso representó inicialmente una dificultad para los cursantes generando ciertos desencuentros iniciales, destacando lo que Ezeiza \& Palacios (2009) muestran, la competencia comunicativa y social de los estudiantes evolucionaría en los foros virtuales al ser utilizados. Aquí encontrarse familiarizado con otro entorno virtual (otro diseño tecnopedagógico) operó en un caso como dificultad por considerar al diseño poco intuitivo. A partir de lo dicho, es posible reconocer la necesidad de la presencia social (Garrison, 2007) que encamine el diálogo más allá de lo estrictamente cognitivo o relacionado con la tarea, aunque sin embargo, quizá existe cierta limitación propia del recurso tecnológico (Coll et al, 2007).

Se destacó, al igual que lo hacen diversos autores, la importancia de tomar decisiones fundamentadas en criterios pedagógicos y en la experiencia y el encontrarse con colegas a fin de reflexionar sobre la propia práctica docente (Aragón, 2013; Onrubia, 2016; Sotomayor, 2016; Sanjurjo \& Rosdríguez, 2003; Szpiniak \& Sanz, 2009) -incluso con colegas provenientes de otras disciplinas-, algo aparentemente poco frecuente en el ámbito local y que excedería el manejo técnico de la plataforma (Borgobello et al, 2018; Copertari et al, 2010).

Las narraciones destacaron la experiencia del taller como una exploración reflexiva, como un aprender haciendo. Tal como se dijo, en esta idea de aprendizaje, diferenciándose de la de ensayo y error, remarcó el sentido de proceso, ensayando y poniendo a prueba, experimentando. En este sentido, se distanciaron de ese clima de agobio por falta de tiempo suficiente mencionado por Montero \& Gewerc (2010) para reflexionar, practicar y evaluar cambios utilizando TIC.

En cuanto al segundo eje de análisis, el rol docente, en los escritos se destacaron ideas que reconocían tradiciones pedagógicas arraigadas en la universidad y otras vinculadas al trabajo con entornos virtuales. En este marco, la evaluación se constituyó en eje de debate cuestionando las tradiciones a partir de la diversidad de recursos que ofrecen los entornos digitales, dando posibilidad, por ejemplo, de seguimiento individualizado o de grupos de estudiantes (García, 2007). Esto puede leerse en un contexto local en el que, a decir de Montero \& Gewerc (2010), las instituciones educativas se encuentran en una situación de tipo dilemática entre su tradicional rol de conservación de la cultura aceptada y las demandas hacia mayor innovación.

Se mencionó en numerosas oportunidades la necesidad de pensar el rol docente en hibridación entre espacios virtuales y cara-a-cara reconociendo las diferencias que presentan en cuanto a la comunicación leída como conflictiva, a la disciplina enseńada y al tipo de tarea en la especificidad de contenidos, entre otros (Borgobello et al, 2010, 2016; Roselli et al, 2010). Esta posibilidad de bimodalidad mostró ciertas dificultades respecto a despegar la idea de rol docente de la presencia cara-a-cara y como demanda en el marco del curso.

En ocasiones el rol docente fue asociado al conjunto de permisos en Moodle relacionado con usuarios diferenciados a partir de distintas asignaciones de edición. Por tanto, apareció como práctica en el ejercicio del rol docente en términos de la especificidad de lo digital (Garrison, 2007). En este sentido y en función de haber sido uno de los ejes del taller desarrollado, el rol docente se leyó como una cierta aplicación contextualizada posible a cursos propios a futuro. Se destacó el trato colaborativo y alentador como parte del rol docente en la coordinación del espacio.

En numerosas oportunidades emergió la idea de docentes con docentes, de la horizontalidad que, si bien suele complicarse en la práctica docente cotidiana, es uno de los ejes del diseño de Moodle (Sotomayor, 2016; Szpiniak \& Sanz, 2009). Así, 
el intercambio de conceptos y experiencias, en palabras de los propios colegas, fue incrementándose en el transcurso del taller. A partir de lo expuesto, la función tutorial en relación al rol docente, segundo eje de análisis, se dio tanto en sentido par como de expertos (Borgobello \& Peralta, 2010). Por tanto, pudo vislumbrarse en la organización de contenidos del programa y tareas (desde la experticia) que progresivamente fueron dando lugar a la autonomía a partir de cierta regulación externa (Roselli, 2016). Sintéticamente, el rol docente apareció vinculado al trabajo con estudiantes en espacios distintos (fundamentalmente y dada la temática del curso: virtual y presencial), a la especificidad del trabajo en el espacio digital, y a las alternancias posibles entre docentes de docentes $y$ docentes con docentes.

El tercer eje de análisis, la categoría tiempo, atravesó las narraciones de manera sistemática, aunque no fue un eje de trabajo del programa del seminario sino un emergente en la lectura de los trabajos y reflexión a posteriori, por lo que se adelantaron en ese apartado discusiones que aquí volvemos a enumerar a modo de cierre. El tiempo fue leído como obstáculo y como posibilitador. En relación a la idea de experiencia, el tiempo se relacionó con la comprensión como procesos favorecidos por el uso de la plataforma (Szpiniak \& Sanz, 2009), con las etapas, con el transcurrir, con el acompañamiento de las docentes responsables del taller (Borgobello et al, 2010; Roselli, 2016), con la familiarización con los recursos y la lógica de la plataforma (Coll et al, 2007), con la motivación por innovar y motivar a los estudiantes; con la distribución paulatina de la presencia docente tornándose trabajo colaborativo entre pares (Garrison, 2007; Sotomayor, 2016, Roselli, 2016), con la formación docente (Acedo, 2017); con la regularidad, fluidez y discontinuidad propio de lo asincrónico (García, 2007); y con la ejercitación con y en el espacio virtual (Borgobello, 2018), en ese aprender haciendo mencionado en los escritos. Podría resumirse este conjunto de ideas que relacionan tiempo y experiencia con cierta resignificación de los procesos de enseñanza y aprendizaje -que exceden el trabajo con entornos virtuales- y que requerirían práctica docente socializada y reflexiva.
Si bien en el trascurso del taller las y los participantes enunciaron cambios pedagógicos factibles producto de la reflexión colectiva, cabe señalar que las afiliaciones institucionales educativas (Pierella, 2017) basadas en clases presenciales con formato tradicional muestran habitualmente resistencia a esos cambios (Aragón, 2013) y docentes que enseñan tal como aprendieron (Ventura, 2016). Por esta razón, sería pertinente investigar qué de esos cambios enunciados logran plasmarse a posteriori de instancias de formación docente en lo cotidiano de los diseños pedagógicos con TIC. En este sentido, tal como señalan Montero \& Gewerc (2010), este aprender como docente desde un rol activo, con colegas y reflexivo para capitalizar ese aprendizaje en la enseñanza, resulta algo agobiante en agendas cargadas de actividades propias del oficio docente que ahora suman las digitales (García, 2007; Sanjurjo \& Rodríguez, 2003; Silva, 2012). A decir de los participantes, a veces resulta difícil de compatibilizar formación y trabajo docente con agendas colectivas.

En coincidencia con Alucin (2018), los escritos analizados sostienen que la incorporación de Moodle propiciaría cambios pedagógicos que resignifican tradiciones universitarias con potencialidad democratizadora, en especial en universidades masivas como las argentinas. Si bien a nivel local se encuentra disponible un campus virtual, tal como sostuvieron los participantes, la incorporación de entornos virtuales en la enseńanza universitaria es aún escasa (Alucin, 2018; Copertari et al, 2010) y su uso suele estar más vinculado a la voluntad de cada docente que a proyectos pedagógicos colectivos (Borgobello, 2018). Además, el soporte técnico suele ser criticado, desestimando potencialmente el uso de TIC (Lorenzo, 2018).

A modo de cierre, destacamos la necesidad de investigaciones y reflexiones colectivas que recuperen en el ejercicio del rol docente ideas de lo colectivo en reflexiones conjuntas, en encuentros con lo digital que, si bien requieren "formación técnica”, pongan el acento en aspectos pedagógicos y contextualizados. 
Análisis cualitativo de una propuesta pedagógica para la incorporación de entornos virtuales a la enseńanza universitaria en Argentina a partir de tres ejes: experiencia, rol docente y tiempo

\section{REFERENCIAS BIBLIOGRÁFICAS}

Acedo, A. (2017). La nueva enseńanza del Derecho en el Espacio Europeo de Educación Superior (EEES). En J. Escobar Gómez (Ed.). Educación Digital y Gestión del Talento Humano en Iberoamérica (pp.68-91). Medellín: CIMTED Corporación.

Achilli, E. (2005). Investigar en Antropología social: los desafíos de transmitir un oficio. Rosario: Laborde.

Alucin, S. (2018). Políticas públicas de educación virtual: Un estado del arte sobre su desarrollo en la UNR. En S.Copertari \& N.Sgreccia (Eds.) Políticas universitarias, comunidades virtuales y experiencias innovadoras en educación (pp.211-244). Rosario: Laborde.

Aragón, M. (2013). Un vistazo a la eficiencia de la plataforma Moodle como recurso de apoyo en unidades de aprendizaje presenciales. Revista Iberoamericana Para La Investigación y El Desarrollo Educativo, 11(1), 1-24.

Borgobello, A. (2018). Realidades mentales y mundos posibles en encuentros pedagógicos presenciales y digitales. En J. Faccendini, P. Martino, M. Sironi, \& M. Terrádez (Eds.), Caleidoscopio. Prácticas y clínicas Psi (pp. 211-224). Rosario: UNR Editora.

Borgobello, A., \& Peralta, N. S. (2010). La función tutorial en el sistema educativo formal: una posible clasificación de sus modalidades. Pensado Psicología, 6(10), 111-121.

Borgobello, A., \& Roselli, N. D. (2016). Rendimiento académico e interacción sociocognitiva de estudiantes en un entorno virtual. Educação e Pesquisa, 42(2), 359-374. https:// doi.org/10.1590/S1517-9702201606143478

Borgobello, A., Sartori, M., \& Roselli, N. D. (2016). ¿Cómo interactuamos aquí y allá? Análisis de expresiones verbales en una clase presencial y otra virtual a partir de dos sistemas de codificación diferentes. Revista de La Educación Superior, 45(179), 95-110. https://doi.org/10.1016/j. resu.2016.06.003

Borgobello, A., Sartori, M., \& Sanjurjo, L. (2018). Concepciones de docentes sobre los estudiantes y sus prácticas pedagógicas. Educación y Educadores, 21(1), 27-48. https://doi. org/10.5294/edu.2018.21.1.2

Coll, C., Onrubia, J., \& Mauri, T. (2007). Tecnología y prácticas pedagógicas: las TIC como instrumentos de mediación de la actividad conjunta de profesores y estudiantes. Anuario de Psicología, 38(3), 377-400.

Copertari, S., Sgreccia, N., \& Fantasía, Y. (2010). Educación a distancia y formación docente. Retos y desafíos en los postgrados de la Universidad Nacional de Rosario. Revista de La Escuela de Ciencias de La Educación, 6(5), 69-88.

Copertari, S., Sgreccia, N., \& Segura, M. L. (2011). Políticas universitarias, Gestión y Formación Docente en Educación a Distancia. Hacia una Pedagogía de la virtualización. Revista de Educación a Distancia, 27(1), 1-16.

Domingo Coscollola, M., \& Fuentes Agustí, M. (2010). Innovación educativa: experimentar con las TIC y reflexionar sobre su uso. Pixel-Bit, 36(1), 171-180.

Echevarría, H. D. (2016). Los diseños de investigación cuantitativa en psicología y educación. Rio Cuarto: UniRio editora.

Ezeiza, A., \& Palacios, S. (2009). Evaluación de la competencia comunicativa y social en foros virtuales. Revista Electrónica de Investigación y Evaluación Educativa, 15(2), 1-15. https://doi.org/10.7203/relieve.15.2.4159

García, A. (2007). Herramientas Tecnológicas Para Mejorar La Docencia Universitaria. Una Reflexión Desde La Experiencia Y La Investigación. RIED, 10(2), 125-148. https:// doi.org/10.5944/ried.2.10.996

Garrison, D. R. (2007). Online community of inquiry review: social, cognitive, and teaching presence issues. Journal of Asynchronous Learning Networks, 11(1), 61-72. https:// doi.org/10.24059/olj.v11i1.1737

Garrison, D. R., \& Kanuka, H. (2004). Blended learning: Uncovering its transformative potential in higher education. The Internet and higher education 7(2), 95-105. https:// doi.org/10.1016/j.iheduc.2004.02.001

Guilar, M. E. (2009). Las ideas de Bruner: "de la revolución cognitiva" a la "revolución cultural.” Educere, 13(44), 235-241.

Lorenzo, J. (2018). Uso de TIC en docentes de nivel medio, terciario y universitario de ciencias humanas y sociales. Anuario Digital de Investigación Educativa, 1(1), 89-104.

Montero, M. L., \& Gewerc, A. (2010). Innovación Posible. Escuelas alteradas por las TIC. Profesorado. Revista de Currículum y Formación de Profesorado, 14(1), 303-318.

Onrubia, J. (2016). Aprender y enseñar en entornos virtuales: actividad conjunta, ayuda pedagógica y construcción del conocimiento. RED, 50(3), 1-16. https://doi.org/10.6018/ $\mathrm{red} / 50 / 3$

Pierella, M. P. (2017). Enseñar en la universidad pública argentina: Los desafíos del oficio docente en una época de transformaciones. Roteiro, 42(1), 37-64. https://doi.org/10.18593/r.v42i1.11548

Piovani, J. (2007). Otras formas de análisis. En A. Marradi, N. Achenti, \& J. Piovani (comp.). Metodología de las Ciencias Sociales (pp.287-298). Buenos Aires: Emecé.

Roselli, N. D. (2016). Los beneficios de la regulación externa de la colaboración sociocognitiva entre pares: ilustraciones experimentales. Revista Puertorriqueña De Psicología, 27(2), 354-367.

Roselli, N. D., Dominino, M., \& Peralta, N. S. (2010). Influencia del tipo de tarea sobre la interacción colaborativa en equipos virtuales. Revista de Psicología General y Aplicada, 63(1), 97-118.

Sanjurjo, L.O. \& Rodríguez, X. (2003). Volver a pensar la clase. Rosario: HomoSapiens.

Sanjurjo, L.O. (2016). La práctica como eje articulador de las propuestas curriculares y didácticas en la formación profesional. En M. Insaurralde (comp.), La enseñanza en la educación superior (pp.195-206). Buenos Aires: Noveduc. 
Silva, J. (2012). Estándares TIC para la Formación Inicial Docente: una política pública en el contexto chileno. Archivos Analíticos de Políticas Educativas, 20(7)7, 1-37. https:// doi.org/10.14507/epaa.v20n7.2012

Sotomayor, G. (2016). Comunidades virtuales de aprendizaje colaborativo para la educación superior. Propósitos y Representaciones, 2(2), 231-303. https://doi.org/10.20511/ pyr2014.v2n2.64

Szpiniak, A. F., \& Sanz, C. V. (2009). Hacia un modelo de evaluación de entornos virtuales de enseńanza y aprendizaje. La importancia de la usabilidad. TE\&ET, 4(1), 10-21.

Ventura, A. C. (2016). ¿Enseño como aprendí?: el rol del estilo de aprendizaje en la enseñanza del profesorado universitario. Aula Abierta, 44(1), 91-98. https://doi.org/10.1016/j. aula.2016.05.001 